preoperative TSH levels were significantly higher in the 241 patients with malignancy ( 2.5 $\pm 0.3 \mathrm{mlU} / \mathrm{l})$ than in the 602 patients with benign disease $(1.4 \pm 0.1 \mathrm{mIU} / \mathrm{l})$. The prevalence of malignancy was lowest in patients with TSH levels $<0.06 \mathrm{mIU} / \mathrm{l}$ and highest in patients with TSH levels $\geq 5.0 \mathrm{mIU} / \mathrm{l}$ (17\% versus $46 \%$ ). The increased risk of DTC in patients with elevated preoperative TSH values persisted when levothyroxine-treated patients were removed from the analysis. An increased risk of DTC was also seen in patients whose TSH levels were within the normal range, but above the population mean. In addition, patients with advanced DTC had significantly higher mean TSH levels than those with low-grade tumors.

The authors conclude that high TSH levels are associated with increased incidence and with advanced stages of DTC, and suggest that TSH could have an important role in the development and progression of thyroid cancer.

Original article Haymart MR et al. (2008) Higher serum thyroid stimulating hormone level in thyroid nodule patients is associated with greater risks of differentiated thyroid cancer and advanced tumor stage. J Clin Endocrinol Metab 93: 809-814

\section{High IGF-I levels imply active acromegaly even when GH levels are 'normal'}

Biochemical control of acromegaly in noncured patients is usually defined as serum growth hormone $(\mathrm{GH})$ levels $<2.0 \mu \mathrm{g} / \mathrm{l}$, plus normalfor-age serum levels of insulin-like growth factor I (IGF-I). Alexopoulou et al., however, have confirmed reports of discordant IGF-I and GH results in noncured patients with acromegaly: in over one-third of their cohort, one parameter was elevated despite the other being normal.

The Belgian Acromegaly Register provided data on 229 patients (mean age \pm SD 54 \pm 14 years; 124 men) with noncured acromegaly. Serum IGF-I and GH levels were measured by validated assays. In total, 81 patients had active disease (IGF-I and GH both high), 68 patients had biochemically controlled disease (IGF-I and GH both normal), and 80 patients had discordant results ( 25 had elevated $\mathrm{GH}$ only, and 55 had elevated IGF-I only). Notably, the high-GH phenotype was most frequent in young, nonirradiated, estrogen-sufficient women, which could indicate roles for age, sex and radiotherapy in IGF-I-GH discordance. The
high-IGF-I phenotype was twice as common as the high-GH phenotype, and was associated with a notably poor metabolic profile; the authors concluded that high IGF-I might indicate persistently active disease even when mean $\mathrm{GH}$ values lie in the normal range.

The authors infer that constant peripheral exposure to even slightly elevated $\mathrm{GH}$ levels might be sufficient to raise IGF-I levels above normal, and suggest that different $\mathrm{GH}$ threshold values might be needed to monitor acromegaly in estrogen-exposed patients.

Original article Alexopoulou O et al. (2008) Divergence between growth hormone and insulin-like growth factor-1 concentration in the follow-up of acromegaly. JClin Endocrinol Metab [doi:10.1210/jc.2007-2104]

\section{DMPA use by young women decreases spine and hip BMD}

Several studies have linked depot medroxyprogesterone acetate (DMPA) use with reduced BMD. Walsh and colleagues' case-control study assessed whether this BMD deficit is age-specific and/or site-specific, and examined the effects of DMPA on markers of bone turnover and hormone levels.

The study included 50 white women aged 18-25 years who began DMPA use before age 20 years (before peak bone mass was attained), and 50 white women aged $35-45$ years who began DMPA use after age 34 years (after peak bone mass was attained). DMPA users were paired with controls matched for age, height, $\mathrm{BMI}$ and smoking status.

Compared with controls, BMD was decreased at the lumbar spine $(-5.6 \%, P<0.05)$ and hip $(-5.2 \%, P<0.05)$ in DMPA users aged 18 25 years only. There was no difference in forearm BMD between DMPA users and controls in either age-group. Regardless of age, serum levels of propeptide of type I procollagen and $\mathrm{N}$-terminal telopeptide of type I collagen (markers of bone turnover) and insulin-like growth factor 1 were significantly higher in DMPA users than controls $(P<0.05)$, whereas levels of estradiol were significantly lower in DMPA users than controls $(P<0.001)$.

The authors conclude that DMPA is associated with a site-specific BMD deficit in users who have not attained their peak bone mass, and that estrogen deficiency is the main mechanism of increased bone turnover. Further 